\title{
Comparison of prospective head motion correction with NMR field probes and an optical tracking system
}

Eschelbach, Martin; Aghaeifar, Ali; Bause, Jonas; Handwerker, Jonas; Anders, Jens; Engel, Eva Maria; Thielscher, Axel; Scheffler, Klaus

Published in:

Magnetic Resonance in Medicine

Link to article, DOI:

$10.1002 / \mathrm{mrm} .27343$

Publication date:

2018

Document Version

Peer reviewed version

Link back to DTU Orbit

Citation (APA):

Eschelbach, M., Aghaeifar, A., Bause, J., Handwerker, J., Anders, J., Engel, E. M., Thielscher, A., \& Scheffler, K. (2018). Comparison of prospective head motion correction with NMR field probes and an optical tracking system. Magnetic Resonance in Medicine, 81(1), 719-729. https://doi.org/10.1002/mrm.27343

\section{General rights}

Copyright and moral rights for the publications made accessible in the public portal are retained by the authors and/or other copyright owners and it is a condition of accessing publications that users recognise and abide by the legal requirements associated with these rights.

- Users may download and print one copy of any publication from the public portal for the purpose of private study or research.

- You may not further distribute the material or use it for any profit-making activity or commercial gain

- You may freely distribute the URL identifying the publication in the public portal 


\section{Comparison of Prospective Head Motion Correction with NMR Field Probes and an Optical Tracking System}

M. Eschelbach ${ }^{1}$, A. Aghaeifar ${ }^{1}$, J. Bause ${ }^{1}$, J. Handwerker ${ }^{2,3}$, J. Anders ${ }^{2,3}$, E.-M. Engel ${ }^{4}$, A. Thielscher ${ }^{1,5,6}$ and K. Scheffler ${ }^{1,7}$

1 Max Planck Institute for Biological Cybernetics, Tuebingen, Germany

2 Institute for Microelectronics, University of Ulm, Ulm Germany

3 Institute of Smart Sensors, University of Stuttgart, Stuttgart, Germany

4 Department of Prosthodontics, Center of Dentistry, Oral Medicine, and Maxillofacial Surgery, University Hospital Tuebingen, Germany

5 Department of Electrical Engineering, Technical University of Denmark, Lyngby, Denmark

6 DRCMR, Copenhagen University Hospital Hvidovre, Hvidovre, Denmark

7 Department for Biomedical Magnetic Resonance, University of Tuebingen, Tuebingen, Germany

Corresponding Author:

Martin Eschelbach, Max-Planck-Ring 11, 72076 Tuebingen, Germany

martin.eschelbach@tuebingen.mpg.de

Running title: Comparison of Prospective Motion Correction with Field Probes and Optical Tracking

Word count: 4315

Key words: head motion; prospective; motion correction; field probes; tracking; Moiré phase tracking 


\section{Abstract}

Purpose

To compare prospective head motion correction and motion tracking abilities of two tracking systems: Active NMR field probes and a Moiré-Phase-Tracking (MPT) camera system using an optical marker.

\section{Methods}

Both tracking systems were used simultaneously on human subjects. The prospective head motion correction was compared in an MP2RAGE and a gradient echo sequence. In addition, the motion tracking trajectories for three subjects were compared against each other and their correlation and deviations were evaluated.

\section{Results}

With both tracking systems motion artifacts were visibly reduced. The precision of the field probe system was on the order of $50 \mu \mathrm{m}$ for translations and $0.03^{\circ}$ for rotations while the camera's was approximately $5 \mu \mathrm{m}$ and $0.007^{\circ}$. The comparison of the measured trajectories showed close correlation and an average absolute deviation below $500 \mu \mathrm{m}$ and $0.5^{\circ}$.

\section{Conclusion}

This study presents the first in vivo comparison between NMR field probes and MPT tracking. For the gradient echo images, the field probes had a similar motion correction performance as the optical tracking system. For the MP2RAGE measurement, however, the camera yielded better results. Thus, the motion tracking modality should be chosen according to the specific requirements of the experiment while considering the desired image resolution, refresh rate and head coil constraints. 


\section{Introduction}

Subject motion is a major problem in functional and anatomical head MRI. The resulting artifacts such as ghosting and blurring may complicate image interpretation, or in the worst case, render acquired images useless. Thus, measurements have to be repeated or entire patient populations, such as elderly or pediatric patients, have to be excluded from certain studies. Furthermore, the high spatial resolution achievable with ultra-high field MRI scanners might be limited by the range of involuntary motion even in trained, cooperative subjects.

Many different methods have been proposed to overcome this obstacle (1). Retrospective motion correction methods (2-4) use the acquired data and try to eliminate motion artifacts with approaches such as image co-registration or entropy based methods (5). While there are manifold powerful retrospective motion correction methods available, inconsistently sampled k-space data and spin-history effects can limit the ability of those methods to eliminate motion artifacts in the images. In addition, especially the co-registration based methods require multiple shots of the same volume to work which is usually only applicable for functional imaging. Prospective motion correction (PMC) on the other hand, uses a motion tracking modality of choice to continuously update the orientation and position of the field of view during data acquisition (6). Those tracking modalities include MR-based methods such as navigators $(7,8)$ or external tracking devices such as NMR markers (9) and optical tracking devices (10). The latter two will be briefly discussed subsequently.

Optical tracking devices using camera(s) have become an established tool in prospective head motion correction. Those devices demonstrated a high accuracy, fast sampling rate and are almost independent from the imaging sequence. However, they require direct line of sight to an optical target or the subject's face (11) and a cross-calibration to convert camera coordinates to scanner coordinates. In addition, they are still expensive, mainly due to the demanding hardware such as a shielded, MR-compatible camera. In contrast, NMR markers such as NMR field probes $(12,13)$ are an alternative that does not require a line of sight and naturally operates in the scanner coordinate system. However, the tracking of NMR markers usually requires additional tracking gradients in all three gradient axes, which can be implemented as a separate gradient block (9), as a modification of imaging gradients (14) or as recently demonstrated, even with the unmodified native imaging gradients (15). NMR field probes have already successfully been used for prospective head motion correction $(14,15)$, but to the authors' knowledge, there has not been any comparison of field probe based prospective motion correction against other methods available. This work compares the prospective head motion correction capabilities of an optical MPT (Moiré-Phase-Tracking) camera-marker system (10) and ${ }^{19} \mathrm{~F}$ NMR field probes (15) in healthy volunteers.

\section{Methods}

\section{Motion Tracking with NMR Field Probes}

The field probe (FP) motion tracking setup consisted of four custom built NMR magnetometers (Figure 1a). Each magnetometer contained a glass capillary tube (inner diameter $0.8 \mathrm{~mm}$ ) filled with 
Hexafluorobenzene $\left(\mathrm{C}_{6} \mathrm{~F}_{6}\right)$ as an NMR active sample and a copper solenoid (inner diameter $1 \mathrm{~mm}$, length $\sim 2 \mathrm{~mm}, 6$ turns) wound around it. To decrease line broadening due to the susceptibility mismatch between air and copper (12), the tubes were embedded in an ellipsoid epoxy casing (radii: $\sim 1.5 \mathrm{~cm}$ and $\sim 1 \mathrm{~cm}$ ) which was susceptibility matched to the copper wire. The probe head was attached to a tuning and matching circuit. It was designed as a transmit/receive system, such that excitation of the sample and signal reception was performed by the same microcoil. The received signals of the FPs were processed by a custom built PCB (Printed Circuit Board) which was connected to the probes via a short $(25 \mathrm{~cm})$ coaxial cable. The PCB hosted a low noise amplifier for the received signal, a power amplifier for transmission and a PLL (Phase Locked Loop) to set the frequency of the transmit RF pulse and to demodulate the received signal - a more detailed description of the electronics can be found in (16). The PCB was powered from an external source outside the scanner room. A standard twisted pair Ethernet cable was used for transmitting all signals from and to the board including the reference frequency for RF transmission and signal demodulation, the trigger for switching between transmit and receive mode and the demodulated field probe signal.

The received demodulated low frequency signal coming from the PCB was digitized by an ADC (National Instruments, Austin, TX, USA) with $2 \mathrm{MS} / \mathrm{s}$ per channel and 16 bit resolution. An in house developed LabVIEW (National Instruments, Austin, TX, USA) program was used to process the digitized data. It extracted the phase from the complex FID signals and also calculated the position of the field probes. The determination of the field probe position in respect to the isocenter of the scanner was realized by switching bipolar gradients on all three axes (Figure 1b). The tracking gradient block of approximately 4 ms was inserted into the sequence after the readout before the excitation pulse of the next TR to minimize $\mathrm{RF}$ interference with the electronics on the board. Each gradient had a length of approximately 1 ms and a strength of $15 \mathrm{mT} / \mathrm{m}$ which was found to be the optimal trade-off between tracking precision and signal dephasing in the field probe sample. Bipolar gradients were chosen to avoid influences of $\mathrm{B}_{0}$ changes, for example due to subject breathing or movement, on the position measurement. A linear fit to the phase of the negative and positive plateau $\dot{\boldsymbol{\varphi}}_{ \pm(r)}$ (see Figure 1c) was applied to determine the gradient amplitudes $\boldsymbol{g}(\boldsymbol{r})$ at a given field probe position $\boldsymbol{r}$. The residual error of the linear fit was used as a measure of the signal quality and to reject noise corrupted measurements. The field probe position $\boldsymbol{r}$ was then calculated from the measured gradient amplitudes as:

$$
\begin{gathered}
\boldsymbol{\omega}_{ \pm(\boldsymbol{r})}=\dot{\boldsymbol{\varphi}}_{ \pm(\boldsymbol{r})}=\gamma \mathbf{1} B_{0}(\boldsymbol{r}) \pm \gamma \boldsymbol{g}(\boldsymbol{r})+\boldsymbol{\eta} \\
\boldsymbol{g}(\boldsymbol{r})=\frac{\dot{\boldsymbol{\varphi}}_{+(\boldsymbol{r})}-\dot{\boldsymbol{\varphi}}_{-(\boldsymbol{r})}}{2 \gamma} \\
\boldsymbol{g}(\boldsymbol{r})=\boldsymbol{G r} \\
\boldsymbol{r}=\boldsymbol{G}^{\mathbf{1}} \boldsymbol{g}(\boldsymbol{r})
\end{gathered}
$$

where $\boldsymbol{\omega}_{ \pm(r)}$ is a vector containing the fitted phase changes acquired during the two gradient lobes at the position $r$ of the field probe. $\gamma$ denotes the gyromagnetic ratio, $1 B_{0}(r)$ the identity matrix and the main magnetic field, $\boldsymbol{\eta}$ the measured noise and $\boldsymbol{G}$ a diagonal matrix with the applied gradient strengths along each axis as diagonal entries. 
Since the assumption of linear gradients does not hold true for actual gradients and would thus decrease the tracking accuracy of the system, a 3rd order spherical harmonics correction based on the scanner vendor's gradient specifications was implemented into the program which generalizes equation 3 to the nonlinear version

$$
\boldsymbol{g}(\boldsymbol{r})=\boldsymbol{G}(\boldsymbol{r}) \boldsymbol{r}
$$

so $\boldsymbol{r}$ can be determined by solving the minimization problem

$$
\boldsymbol{r}=\underset{\boldsymbol{r}}{\arg \min }(\boldsymbol{G}(\boldsymbol{r}) \boldsymbol{r}-\boldsymbol{g}(\boldsymbol{r})),
$$

with a starting value of $\boldsymbol{r}$ calculated from the linear equation 4. Besides that, no further calibration or gradient characterization was implemented to determine the field probe positions. The calculated field probe positions were used to derive the translation of the field probes' geometric center and the rotation using an algorithm proposed by Umeyama (17). The data acquisition and processing including the position calculation for four field probes took approximately $70 \mathrm{~ms}$.

Previous experiments have already evaluated the precision (18) and long term stability of motion tracking with the used field probe setup. The tracking precision, defined as the standard deviation of the tracking noise, for translational motion was measured to be $60 \mu \mathrm{m}, 64 \mu \mathrm{m}$ and $54 \mu \mathrm{m}$ along the $x$-, $y$ - and z-axis, respectively, while the rotation could be resolved to a precision of $0.026^{\circ}$ (average standard deviation). Long term stability experiments revealed a drift of $71 \mu \mathrm{m}$ and $0.006^{\circ}$ over a time span of approximately 50 minutes which is in the same order of magnitude as the precision and in principle also allows the usage in scans with longer acquisition times. The precision values for NMR field probes found in recent literature (14) are even lower and amount to $6.1 \mu \mathrm{m}, 6.3 \mu \mathrm{m}, 10.5 \mu \mathrm{m}$ along $\mathrm{x}, \mathrm{y}$ and $\mathrm{z}$, respectively, as well as $0.003^{\circ}$, $0.004^{\circ}, 0.004^{\circ}$ for rotation around $\mathrm{x}$-, $\mathrm{y}$ - and z-axis, respectively, at a field strength of $7 \mathrm{~T}$.

\section{MPT Motion Tracking}

The optical tracking system is commercially available (Kineticor Inc, HI, USA), and consists of a camera attached to the top of the scanner bore (Figure $2 \mathrm{~b}$ ) and a reflective $15 \times 15 \mathrm{~mm}^{2}$ moire phase tracking (MPT) marker (Figure 2a). This setup allows the estimation of all 6 motion parameters with a single camera with a frame-rate of $85 \mathrm{~Hz}$ (for details see $(10,19)$ ).

The precision of the MPT tracking system according to literature (10) is $1 \mu \mathrm{m}, 12 \mu \mathrm{m}$ and $1 \mu \mathrm{m}$ in the scanner $x, y$ and $z$ directions, respectively and better than $0.01^{\circ}$ in all three rotations which was approximately confirmed in our own experiments (18) with results of $6 \mu \mathrm{m}, 2 \mu \mathrm{m}, 5 \mu \mathrm{m}$ and $0.006^{\circ}, 0.008^{\circ}$, $0.006^{\circ}$, respectively.

Since the camera detects marker motion in its own coordinate system, a cross-calibration is needed to convert the measured data from camera coordinates to the scanner coordinate system. The calibration procedure involves moving a structured phantom with a rigidly attached MPT marker. The motion in scanner coordinates is calculated with a co-registration method while the same motion is also recorded with the camera. With at least three different phantom positions, it is possible to calculate the crosscalibration (20). With more motion repetitions this cross-calibration can be further refined. 
Both systems, field probes and camera based motion tracking, use an external PC that sends the calculated motion parameters to the scanner via User Datagram Protocol (UDP). There, it is processed by a custom built library (21) which has a modular design in order to adapt to different motion tracking sources. The input is then used to calculate the update of the field of view position and orientation accordingly, while always assuming rigid body motion with six degrees of freedom, which was assumed to be sufficient for head motion correction.

Motion Stage Experiment

The MR scanner used in all experiments was a 9.4 T human scanner (Siemens Healthcare, Erlangen, Germany) equipped with a whole body gradient system (SC72).

A custom built motion stage was used to assess the accuracy of the motion tracking of both systems. The motion stage consisted of a sledge that hosted the MPT marker and a field probe (Figure 3 ). The sledge was mounted onto a threaded rod with an attached MR-compatible sliding caliper to measure the distance traveled by the sledge. The fine scale of the caliper was used to acquire position measurements in $500 \mu \mathrm{m}$ steps. The MPT marker was moved concurrently with a field probe over a total distance of $3 \mathrm{~cm}$ and back by turning the screw of the positioning stage inside the scanner bore. For each motion step the position was recorded simultaneously with the field probe and the MPT camera. The starting point of the measurement was at around $+16 \mathrm{~cm}$ off-isocenter in z-direction with direction of the motion approximately along positive $z$. This position was chosen since it is far-off enough from the isocenter for non-linear effects of the gradients to show in the field probe measurements.

\section{In Vivo Experiments}

Three healthy volunteers (male, $26-38$ y) were measured in accordance to the local ethics protocol using a custom built $16 \mathrm{Tx} / 31 \mathrm{Rx}$ head coil (22). The MPT marker was attached to the subjects on a custom built bite-bar, specifically designed to fit the unique dental impression of each subject (Fig. 2a). This ensures a fairly rigid coupling to any movements of the skull and free line of sight of the camera to the marker despite the closed coil cover. The field probes were attached to the nose bridge and the temples of the subjects using medical tape to minimize the effect of skin movement on the motion measurement. An additional mount for the field probes could not be used due to spatial constraints of the receive array.

To compare prospective motion correction with both modalities, three subjects were scanned with a 2D GRE sequence (matrix: $400 \times 400$, resolution: $0.5 \times 0.5 \times 1.6 \mathrm{~mm}^{3}$, slices: 10 , slice spacing: $9.6 \mathrm{~mm}, \mathrm{FA}: 15^{\circ}$, TA: 1:21 min, TR: $160 \mathrm{~ms}$, TE: $9 \mathrm{~ms}$ ). Each subject was asked to perform the following motion patterns: no motion, small slow motion, small fast motion and big motion. Images for each motion pattern were acquired once with no correction, field probe correction and MPT correction, respectively. The sequence motion update was performed every k-space line for the MPT correction but only every fourth line for the field probe correction due to the calculation time needed to process the field probe data.

For all GRE measurements, no matter whether the prospective correction was enabled or disabled, the motion trajectories were recorded simultaneously with both tracking modalities in order to compare the 
recorded trajectories against each other. For the tracking comparison, clear outliers in the motion tracking $\left(>2 \mathrm{~mm} / 2^{\circ}\right.$ between successive tracking steps) were removed during post processing.

Additionally, two subjects were scanned with a whole-brain MP2RAGE (23) sequence (TR-FOCl inversion (24)), resolution: $0.55 \mathrm{~mm}$ isotropic, matrix: $384 \times 384 \times 512, \mathrm{FA}_{1 / 2}: 4^{\circ} / 8^{\circ}, \mathrm{Tl}_{1 / 2}: 730 / 1950 \mathrm{~ms}$, TR: $6 \mathrm{~ms}$, MP2RAGE TR: $5000 \mathrm{~ms}$, TE: $3 \mathrm{~ms}$, GRAPPA: 2x2, 6/8 partial Fourier in secondary phase encoding direction, TA: $9.03 \mathrm{~min})$. The MP2RAGE images were acquired with three settings: no correction, field probe correction and MPT correction and the subject being instructed to hold still. The motion update for both, MPT and field probe correction, was performed only before each inversion pulse (every $5 \mathrm{~s}$ ). In addition, an actual flip angle map ((25), $3.3 \mathrm{~mm}$ isotropic, FA: 50', TR1/2: 20/100 ms, GRAPPA: 2×2, TA: 1:20 min) was acquired. This allowed to correct for residual $\mathrm{B}_{1}{ }^{+}$influences caused by the high flip-angle chosen for the MP2RAGE measurements by using the approach described by Hagberg et al (26).

\section{Results}

\section{Motion Stage}

The measured total displacement during the motion stage experiment of the respective tracking system is depicted in Figure 4a. The trajectories are very similar but the measured final displacement of $30.08 \mathrm{~mm}$ for the MPT tracking and $29.33 \mathrm{~mm}$ for the field probe yield a difference of $750 \mu \mathrm{m}$ between both methods. The differences to the ground truth are $80 \mu \mathrm{m}$ and $670 \mu \mathrm{m}$, respectively. While the former is within the precision of the motion stage gauge, the latter likely represents a mismatch of the measured value to the ground truth. In addition, the effect of the correction of gradient nonlinearities is plotted in the uncorrected trajectory of the field probes. Figure $4 \mathrm{~b}$ shows the displacement difference between successive steps. Here, the measurements of both modalities stay within the motion stage's precision. The difference between both tracking modalities for the measured displacement steps is shown in Figure 4c. The mean absolute difference between both systems amounted to $21 \mu \mathrm{m}$.

\section{Motion Tracking Comparison}

For a quantitative comparison of the measured trajectories, all motion data-points of the in vivo GRE measurements were compared against each other. . Tracking data from 36 scans was used to create a scatter plot of field probe tracking versus MPT tracking (Figure 5). The scatter plot shows again the underestimation of translational motion for the field probes compared to the MPT tracking which was already visible in the results of the motion stage experiment (Figure 4). Table 1 summarizes the comparison of the two tracking modalities. The motion range covered by the experiments is a few millimeters in translational motion, with maximum values of $-2.59 \mathrm{~mm}$ up to $+3.68 \mathrm{~mm}$ in $\mathrm{x}$-direction and several degrees of rotational motion with a minimum of $-4.95^{\circ}$ and a maximum of $+4.17^{\circ}$, both around the $z$-axis. The mean absolute deviation is well below $1 \mathrm{~mm} / 1^{\circ}$ for all degrees of freedom even when including the corresponding standard deviations. However, the maximum absolute deviation for the two systems was 
$2.02 \mathrm{~mm}$ along $\mathrm{z}$ and $0.9^{\circ}$ around the z-axis. In addition to the slopes calculated from the linear fits in Figure 5, the table also shows the Pearson correlation coefficient PCC which provides a measure for correlation between two variables with values between -1 and 1 with 1 representing perfect correlation, -1 negative correlation and 0 no detectable correlation at all. The coefficients show positive correlation with a correlation coefficient above 0.9 for all degrees of freedom other than the translations in $y$ and $z$ where the correlation coefficient is only 0.61 and 0.79 , respectively.

\section{D GRE}

An exemplary slice of the acquired 2D GRE images of one subject for all twelve imaging conditions along with sample motion trajectories for each motion condition is depicted in Figure 6 . The images without voluntary motion show the consistency across all three imaging conditions. There is no visible decrease in image quality when the prospective motion correction is enabled (top row in Figure 6). The images acquired with voluntary subject motion (rows 2-4 in Figure 6) clearly show motion artifacts in the uncorrected case which are substantially reduced when enabling prospective motion correction with either tracking modality. However, the quality of the corrected images is still a little decreased compared to the images without voluntary motion. This is especially true when looking at images for the case of big motion where remaining motion artifacts are still visible for both correction modalities.

One sample trajectory for both tracking modalities corresponding to the 2D GRE measurement with field probe correction and big motion is shown in Figure 7. The lower precision of the field probe system is clearly visible in its noisier data. Once more, there is a visible tendency of underestimation of translational motion in the field probe data versus the camera measurements, where translations in z-direction show the least correlation between the two tracking systems. In addition, there is a sudden jump in $\mathrm{Tz}$ after approximately $40 \mathrm{~s}$. But there are also a few data points missing for the camera measurement. However, when comparing the measured rotational motion both trajectories match well.

\section{MP2RAGE}

Figure 8 shows a slice for the MP2RAGE measurements in one subject using three different motion correction settings. The range of motion in all three measurements was very similar as shown in the corresponding motion trajectories captured by the MPT camera system. The images for the second subject are not shown because the captured motion range varied more between the three scans. In the displayed measurements, upon closer inspection, the field probe corrected image shows an improvement over the uncorrected case but does not quite reach the quality of the MPT corrected image. The final head position during the measurement with FP correction deviated by $-0.32 \mathrm{~mm},-0.34$ $\mathrm{mm}$ and $-0.50 \mathrm{~mm}$ along $\mathrm{x}, \mathrm{y}$, and $\mathrm{z}$, respectively, and $-0.49^{\circ},-0.14^{\circ}$ and $0.24^{\circ}$ around $\mathrm{x}, \mathrm{y}$ and $\mathrm{z}$, respectively, from the simultaneously acquired camera data.

\section{Discussion}


The motion stage experiment showed that the displacements between successive steps determined with the field probes agreed very well with the measurements from the camera as well as with the ground truth from the motion stage. However, for large motion and positions of the field probes far away from the isocenter, the $3^{\text {rd }}$ order nonlinearity correction does not seem to be sufficient anymore. But with the field probes usually being closer to the isocenter when attached to the head and translations of $3 \mathrm{~cm}$ being an example of extreme motion, in most usage scenarios this won't affect the prospective correction to a large extend. But this still shows that an accurate gradient characterization for the exact position determination of the field probes remains crucial and gradient characterization beyond the vendor's specifications, e.g. by measuring its full impulse response function (27), might further improve the accuracy of the tracking results. However, such measurements would ideally require a field camera which was not available for this study.

The results of the motion tracking comparison revealed a close agreement in measured rotational motion while translational motion tended to be underestimated by the field probes in comparison to MPT tracking. A reason for the observed mismatch in translational motion especially along the z-axis might be that the z-position of the field probes was measured with the last bipolar gradient. At this time point there is less signal available from the field probe sample than at the beginning of the FID acquisition. The observed low correlation for the Ty measurements is probably due to the relatively small range of motion of the subjects along that axis. This is also connected to the limited space available for head motion inside the receive helmet.

However, part of the discrepancy might also stem from residual movement of the field probes in respect to the skull. Since both motion tracking methods are marker based, a rigid attachment of the respective marker(s) is a crucial factor in influencing the accuracy and stability of the measurements. Therefore, an attachment of the field probes to the bite-bar has also been tested. But in this configuration the inherent noise of the field probe position measurements was leveraged by the longer distance to the imaging region. Thus it was found to be the best solution if the field probes were placed in close proximity to the brain. Another possible source of errors was the remaining noise in the field probe measurements that lead to a decreased precision in the determination of their position. Parts of the noise were already removed by demodulating the signal in the scanner and low-pass-filtering the demodulated signal before digitization as well as rejecting some of the noisy measurements. However, further improvements may be achieved by increasing the sample size of the field probes or employing short-lived probes (15) in order to increase SNR. In contrast to the field probes, the camera accuracy mainly depends on the quality of the cross-calibration (28) and invalid measurements were typically caused by reflections on the marker itself.

The motion corrected 2D GRE images show the ability of both, the MPT system and the field probe based motion correction, to substantially reduce motion artifacts in the presence of subject motion. The tracking differences especially for measured translational motion are not visibly translated in a decreased image quality. One reason might be that natural head motion mainly consists of rotations while translational motion tends to be small. Also, the mean absolute deviation between camera and field probes for the translational motion (see Table 1) was still below half the voxel size. The displayed motion trajectory (Fig. 7) shows that the general motion pattern in terms of magnitude and direction is very similar for both tracking modalities. However, the short jump of the Tz-trajectory after $\sim 40 \mathrm{~s}$ may be caused by a field probe hitting the receive helmet, since it coincides with the time point of maximal head rotation around $z$ in that measurement. 
For the MP2RAGE measurements the improvement in image quality for both correction methods is clearly visible. Here, however, the camera corrected images show a higher quality than the ones corrected with the field probes. During the longer measurement it is more likely for the field probes' locations to move due to non-rigid attachment to the skin. But still the calculated tracking deviation between the two systems for the final head position was only in the order of a voxel size. Overall, the field probe measurements still proved sufficient to substantially increase image quality compared to the uncorrected case.

Since all the measurements were carried out at a field strength of 9.4T one might argue that there is an SNR advantage which might increase the precision of the field probe measurements compared to experiments at scanners with a lower field strength. But it has been shown that a comparable precision can be also attained at 3T systems (14).

One drawback of our implementation of field probe motion correction is the necessity of altering the sequence and the need for additional time in each TR for implementing the tracking gradients as well as for processing the field probe data. But as recent work has shown, it is also possible to use the imaging gradients for encoding the field probe position (15). The processing time could possibly be decreased further by outsourcing parts of the LabVIEW code to an implementation with a faster programming language.

Advantages of the field probes include the lack of a cross-calibration procedure and the fact that no line of sight to a target is needed, thus there is no need for a bite-bar even in closed coil setups. This increases subject comfort according to the subjects participating in this study. On the other hand, one still has to attach the field probes to the subject's head which again reduces comfort and currently remains a time consuming procedure, therefore still preventing an application in clinical environments. In addition, the signal transmission and power supply to the PCBs still requires a set of cables. Moving to wireless (29) or optical transmission would increase the applicability in a clinical setup.

In conclusion, even though the inherent precision of our implementation of field probe based tracking is one order of magnitude worse than the MPT camera systems' (approx. $50 \mu \mathrm{m}$ vs $5 \mu \mathrm{m}, 0.03^{\circ}$ vs $0.007^{\circ}$ ), for an imaging resolution well above the precision limit (voxel size $0.5 \times 0.5 \times 1.6 \mathrm{~mm}^{3}$ ), both systems performed similarly well regarding the quality of the motion corrected 2D GRE images. For the MP2RAGE measurements however the MPT system performed better probably due to field probe motion during the longer acquisition time. While there is still room for improvement in the precision and calculation time of NMR field probe tracking, the most crucial point for reliable prospective head motion correction remains to be the rigid attachment of the respective tracking markers to the skull. If this is taken into account, field probes can be a suitable alternative to optical tracking systems especially when considering recent advances in precision and the use of imaging gradients for position encoding (15). In addition, there are further possible applications of field probes, such as field monitoring (30) or collecting physiological data (31), which could be obtained from the concurrently measured $B_{0}$ component of the signal.

\section{Acknowledgements}

This work was supported by the Deutsche Forschungsgemeinschaft (DFG) grants SCHE658/4 and TH1330/5.

\section{References}


1. Godenschweger F, Kägebein U, Stucht D, Yarach U, Sciarra A, Yakupov R, Lüsebrink F, Schulze P, Speck O. Motion correction in MRI of the brain. Phys. Med. Biol. 2016;61:R32-R56.

2. Morgan VL, Dawant BM, Li Y, Pickens DR. Comparison of $\mathrm{fMRI}$ statistical software packages and strategies for analysis of images containing random and stimulus-correlated motion. Comput. Med. Imaging Graph. 2007;31:436-446.

3. Batchelor PG, Atkinson D, Irarrazaval P, Hill DLG, Hajnal J, Larkman D. Matrix description of general motion correction applied to multishot images. Magn Reson Med 2005;54:1273-1280.

4. Atkinson D, Hill DLG, Stoyle PNR, Summers PE, Clare S, Bowtell R, Keevil SF. Automatic compensation of motion artifacts in MRI. Magn Reson Med 1999;41:163-170.

5. Loktyushin A, Nickisch H, Pohmann R, Schölkopf B. Blind retrospective motion correction of MR images. Magn Reson Med 2013;70:1608-18.

6. Maclaren J, Herbst M, Speck O, Zaitsev M. Prospective motion correction in brain imaging: A review. Magn Reson Med 2013;69:621-36.

7. Van Der Kouwe AJW, Benner T, Dale AM. Real-time rigid body motion correction and shimming using cloverleaf navigators. Magn Reson Med 2006;56:1019-1032.

8. Julian Maclaren, Andre Kyme, Murat Aksoy, Benjamin Zahneisen and RB. Markerless Optical Tracking for Motion Correction in MR and PET/MR Imaging of the Brain. In Proceedings of the 25th Annual Meeting of ISMRM, Honolulu, 2017. p1292.9. Welch EB, Manduca A, Grimm RC, Ward HA, Jack Jr. CR. Spherical navigator echoes for full 3D rigid body motion measurement in MRI. Magn Reson Med 2002;47:32-41.

10. Ooi MB, Krueger S, Thomas WJ, Swaminathan S V, Brown TR. Prospective real-time correction for arbitrary head motion using active markers. Magn Reson Med 2009;62:943-54.

11. Maclaren J, Armstrong BSR, Barrows RT, et al. Measurement and correction of microscopic head motion during magnetic resonance imaging of the brain. PLoS One 2012;7:e48088.

12. De Zanche N, Barmet C, Nordmeyer-Massner J a, Pruessmann KP. NMR probes for measuring magnetic fields and field dynamics in MR systems. Magn Reson Med 2008;60:176-86.

13. Barmet C, De Zanche N, Wilm BJ, Pruessmann KP. A transmit/receive system for magnetic field monitoring of in vivo MRI. Magn Reson Med 2009;62:269-76.

14. Haeberlin M, Kasper L, Barmet C, Brunner DO, Dietrich BE, Gross S, Wilm BJ, Kozerke S, Pruessmann KP. Real-time motion correction using gradient tones and head-mounted NMR field probes. Magn Reson Med 2015;74(3):647-60.

15. Aranovitch A, Haeberlin M, Gross S, Dietrich BE, Wilm BJ, Brunner DO, Schmid T, Luechinger R, Pruessmann KP. Prospective motion correction with NMR markers using only native sequence elements. Magn Reson Med 2017. doi: 10.1002/mrm.26877 
16. Handwerker J, Eschelbach M, Chang P, Henning A, Scheffler K, Ortmanns M, and Anders J. An active TX/RX NMR probe for real-time monitoring of MRI field imperfections. Biomedical Circuits and Systems Conference (BioCAS), 2013 IEEE, 2013, pp. 194-197.

17. Umeyama S. Least-Squares Estimation of Transformation Parameters Between Two Point Patterns. IEEE Trans. Pattern Anal. Mach. Intell. 1991;13:376-380.

18. Eschelbach M, Loktyushin A, Chang P, Handwerker J, Anders J, Henning A, Thielscher A, Scheffler K. A Comparison of 19F NMR Field Probes and an Optical Camera System for Motion Tracking. In Proceedings of the 24th Annual Meeting of ISMRM, Singapore, 2016. p. 0340.

19. Stucht D, Danishad KA, Schulze P, Godenschweger F, Zaitsev M, Speck O. Highest resolution in vivo human brain MRI using prospective motion correction. PLoS One 2015;10:1-17.

20. Zahneisen B, Lovell-Smith C, Herbst M, Zaitsev M, Speck O, Armstrong B, Ernst T. Fast noniterative calibration of an external motion tracking device. Magn Reson Med 2013;1500:1489-1500.

21. Ali Aghaeifar, Martin Eschelbach, Jonas Bause, Axel Thielscher KS. AMoCo, a software package for prospective motion correction. In Proceedings of the 25th Annual Meeting of ISMRM, Honolulu, 2017. p. 305.

22. Shajan G, Kozlov M, Hoffmann J, Turner R, Scheffler K, Pohmann R. A 16-channel dual-row transmit array in combination with a 31-element receive array for human brain imaging at 9.4 T. Magn Reson Med 2013;71(2):870-879.

23. Marques JP, Kober T, Krueger G, van der Zwaag W, Van de Moortele PF, Gruetter R. MP2RAGE, a self bias-field corrected sequence for improved segmentation and T1-mapping at high field. Neuroimage 2010;49:1271-1281.

24. Hurley AC, Al-Radaideh A, Bai L, Aickelin U, Coxon R, Glover P, Gowland PA. Tailored RF pulse for magnetization inversion at ultrahigh field. Magn Reson Med 2010;63:51-8.

25. Yarnykh VL. Actual flip-angle imaging in the pulsed steady state: a method for rapid threedimensional mapping of the transmitted radiofrequency field. Magn Reson Med 2007;57:192-20

26. Hagberg G, Bause J, Ethofer T, et al. Whole brain MP2RAGE-based mapping of the longitudinal relaxation time at 9.4T. Neuroimage 2016;144:203-216.27. Vannesjo SJ, Haeberlin M, Kasper L, Pavan M, Wilm BJ, Barmet C, Pruessmann KP. Gradient system characterization by impulse response measurements with a dynamic field camera. Magn Reson Med 2013;69:583-93.

28. Zahneisen B, Keating B, Ernst T. Propagation of Calibration Errors in Prospective Motion Correction Using External Tracking. Magn Reson Med 2014;72(2):381-388.

29. Ooi MB, Aksoy M, Maclaren J, Watkins RD, Bammer R. Prospective motion correction using inductively coupled wireless RF coils. Magn Reson Med 2013;70(3):639-647. 
30. Vionnet L, Aranovitch A, Duerst Y, Haeberlin M, Dietrich B, Gross S, Lars K, Schmid T, Pruessmann K. Simultaneous Prospective Motion Correction and Feedback Field Control: T2* Weighted Imaging at High Field. In Proceedings of the 25th Annual Meeting of ISMRM, Honolulu, 2017, 306.

31. Gross S, Vionnet L, Kasper L, Dietrich BE, Pruessmann KP. Physiology recording with magnetic field probes for fMRI denoising. Neuroimage 2017;154:106-114.

\section{Figures and Tables}

Figure 1: a) ${ }^{19} \mathrm{~F}$ NMR Field probe with tuning/matching-circuitry and PCB for signal processing. b) Bipolar gradients used for position tracking of the field probes. c) Corresponding measured phase and FID for one field probe when applying three orthogonal bipolar gradients.

Figure 2: a) MPT marker on a custom made bite-bar. b) Positioning of the MPT tracking camera and the field probes in the scanner bore for a closed coil setup.

Figure 3: Motion stage. The sledge with the MPT marker and an NMR field probe can be moved by turning a screw while obtaining exact position information from the caliper.

Figure 4: Results of the motion stage experiment. (A) Measured distance from starting point (approx. $+16 \mathrm{~cm}$ from isocenter along z) for both tacking systems. (B) Measured distance between single motion steps. (C) Difference of field probe and MPT measurements for the distance between single steps.

Figure 5: Scatter plots of all 6 degrees of freedom for in vivo motion tracking (3 subjects, 36 measurements). Field probe tracking against MPT tracking with linear fit and the red line indicating identity.

Figure 6: 2D Gradient echo images with an in-plane resolution of $0.5 \mathrm{~mm} \times 0.5 \mathrm{~mm}$ and a through-plane resolution of $1.6 \mathrm{~mm}$. Zoomed images for all twelve imaging conditions in one subject along with sample motion trajectories measured with the field probes for each motion condition. The full slice view of the measurements can be found in Supporting Figure S1. The motion trajectories for all twelve measurements are depicted in Supporting Figure S2.

Figure 7: Motion trajectory for both tracking modalities from the 2D GRE measurement with big motion and field probe correction in Figure 6. 
Figure 8: MP2RAGE (0.5 $\mathrm{mm}^{3}$, acquisition time 9:03 $\mathrm{min}$ ) images for three motion correction conditions with corresponding motion trajectories acquired with the camera.

Supporting Figure S1: Full slice view for all twelve imaging conditions for the 2D GRE measurements in Fig. 6.

Supporting Figure S2: Motion trajectories captured by the field probes for all twelve motion and correction combinations for the 2D GRE measurements in Fig. 6.

Table 1: Motion range (MPT values), maximum absolute difference, mean absolute deviation (MAD), mean squared deviation (MSD) and corresponding standard deviations $(\sigma)$ as well as the slopes from Fig. 5 and the calculated Pearson correlation coefficients (PCC) for field probes against MPT tracking for the motion experiments across 3 subjects (36 measurements).

$\begin{array}{ccccccc} & \text { Motion Range } & \text { Max Abs Diff } & \text { MAD } \pm \sigma & \text { MSD } \pm \sigma & \text { Slope } & \text { PCC } \\ \text { Tx }[\mathrm{mm}] & -2.59-3.68 & 2.02 & 0.23 \pm 0.21 & 0.10 \pm 0.17 & 0.81 & 0.92 \\ \text { Ty }[\mathrm{mm}] & -0.78-1.30 & 1.08 & 0.12 \pm 0.10 & 0.03 \pm 0.04 & 0.44 & 0.61 \\ \mathbf{T z}[\mathbf{m m}] & -1.78-1.39 & 1.26 & 0.20 \pm 0.15 & 0.06 \pm 0.10 & 0.84 & 0.79 \\ \mathbf{R x}\left[^{\circ}\right] & -2.85-3.81 & 0.89 & 0.14 \pm 0.13 & 0.04 \pm 0.07 & 0.96 & 0.97 \\ \mathbf{R y}\left[^{\circ}\right] & -3.63-2.12 & 0.78 & 0.07 \pm 0.05 & 0.01 \pm 0.02 & 0.97 & 0.99 \\ \mathbf{R z}\left[^{\circ}\right] & -4.95-4.17 & 0.90 & 0.10 \pm 0.11 & 0.02 \pm 0.06 & 0.99 & 0.99\end{array}$




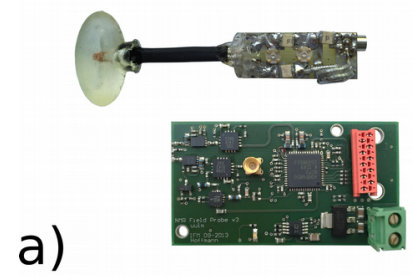

a)

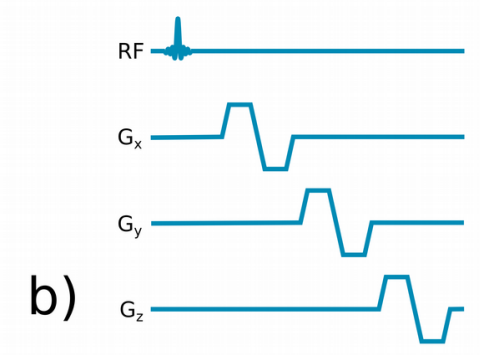

c)

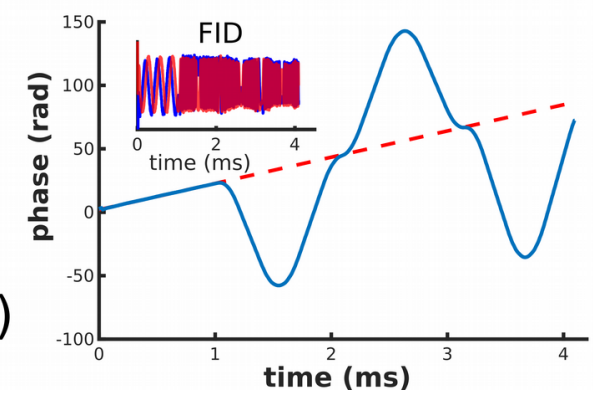

Figure 1: a) ${ }^{19} \mathrm{~F}$ NMR Field probe with tuning/matching-circuitry and PCB for signal processing. b) Bipolar gradients used for position tracking of the field probes. $\mathrm{c}$ ) Corresponding measured phase and FID for one field probe when applying three orthogonal bipolar gradients.

a)
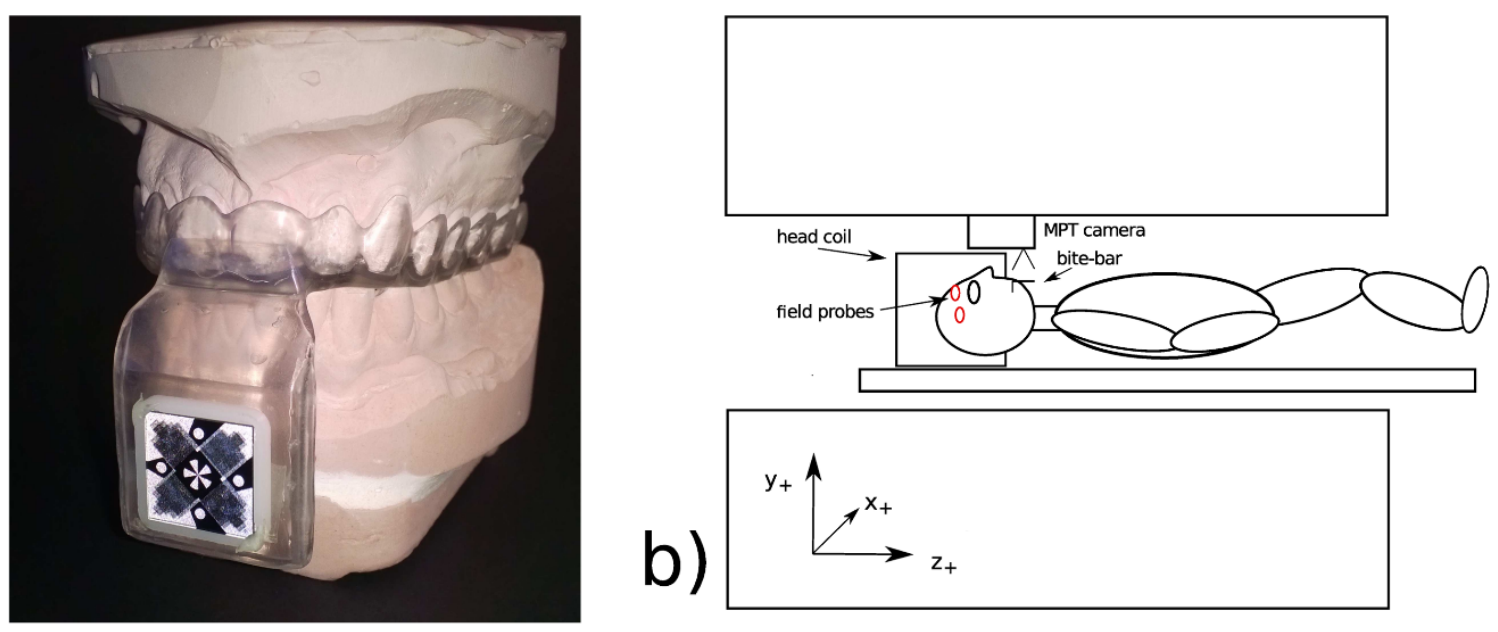

Figure 2: a) MPT marker on a custom made bite-bar. b) Positioning of the MPT tracking camera and the field probes in the scanner bore for a closed coil setup.

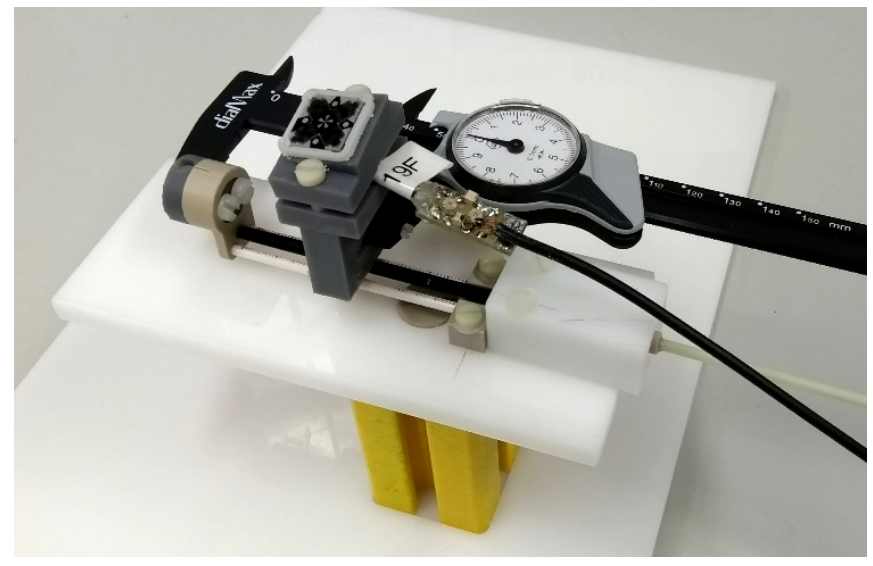

Figure 3: Motion stage. The sledge with the MPT marker and an NMR field probe can be moved by turning a screw while obtaining exact position information from the caliper. 

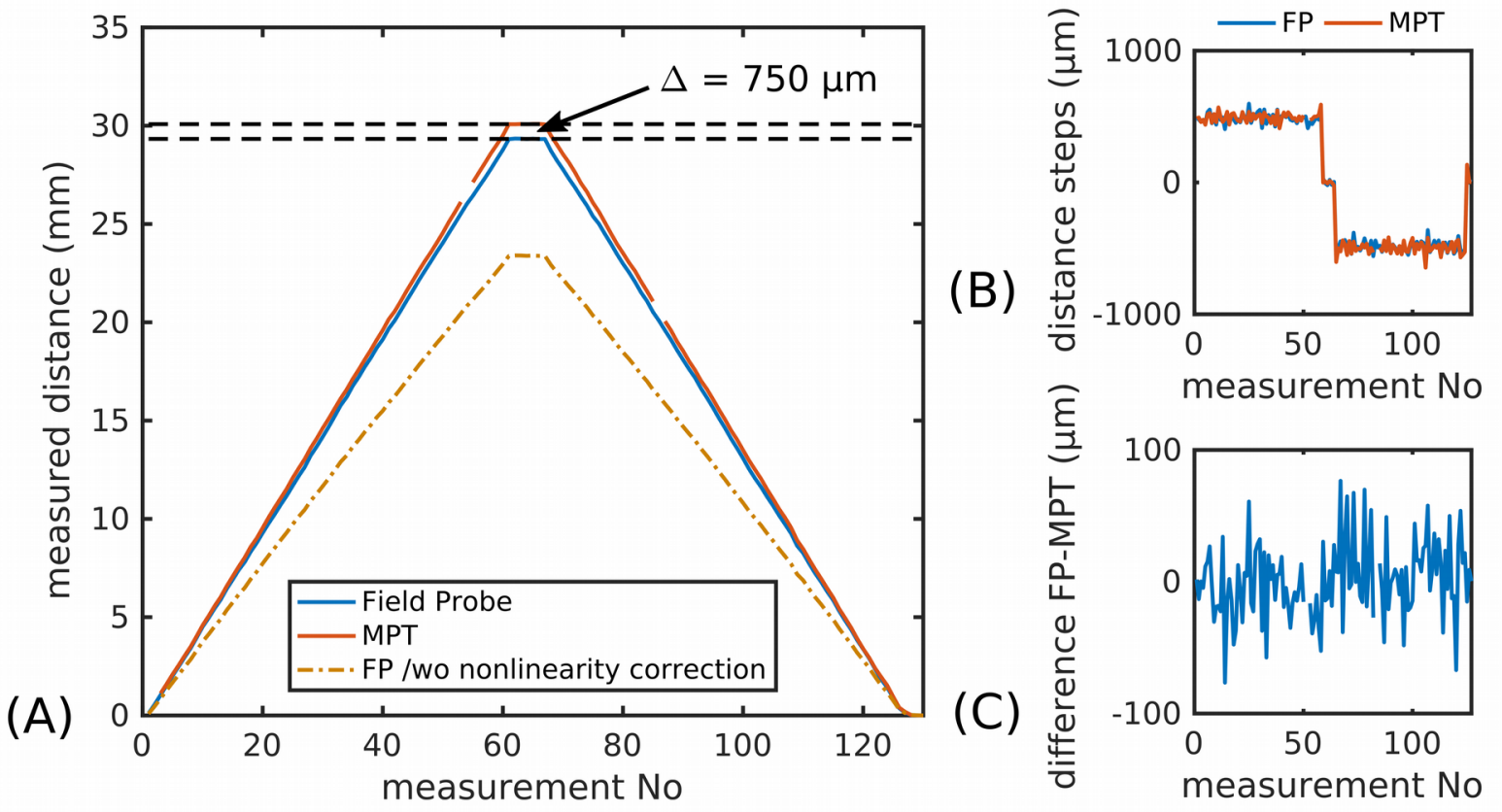

Figure 4: Results of the motion stage experiment. (A) Measured distance from starting point (approx. +16 $\mathrm{cm}$ from isocenter along z) for both tracking systems. (B) Measured distance between successive motion steps. (C) Difference of field probe and MPT measurements for the distance between successive steps.
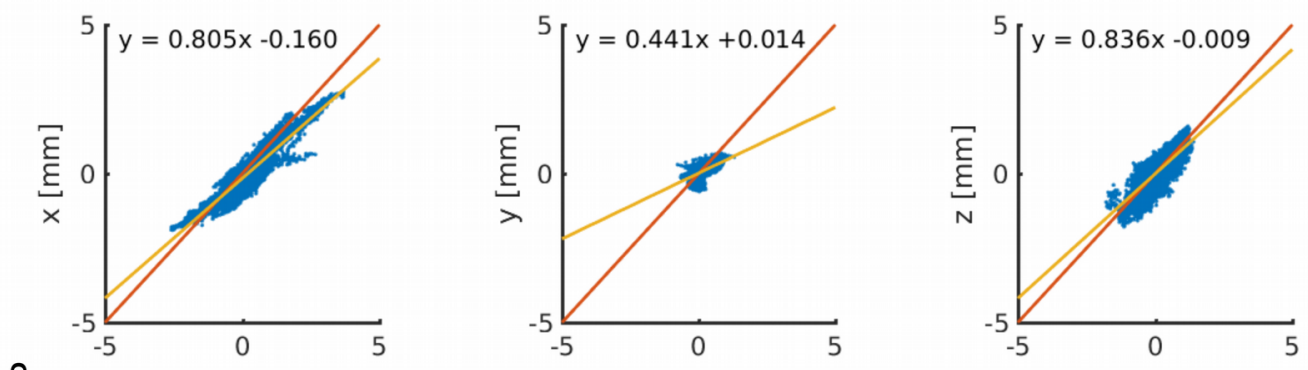

은
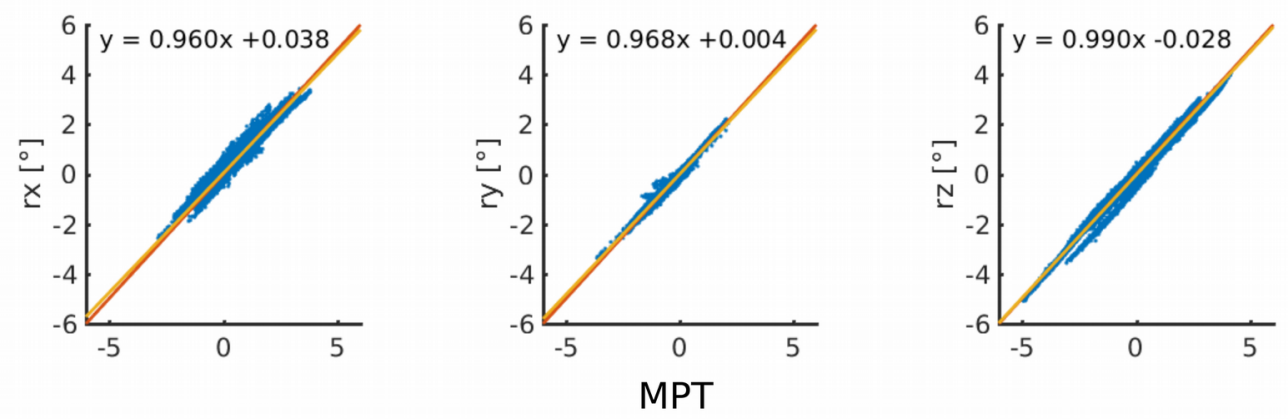

Figure 5: Scatter plots of all 6 degrees of freedom for in vivo motion tracking (3 subjects, 36 measurements). Field probe tracking against MPT tracking with linear fit and the red line indicating identity. 


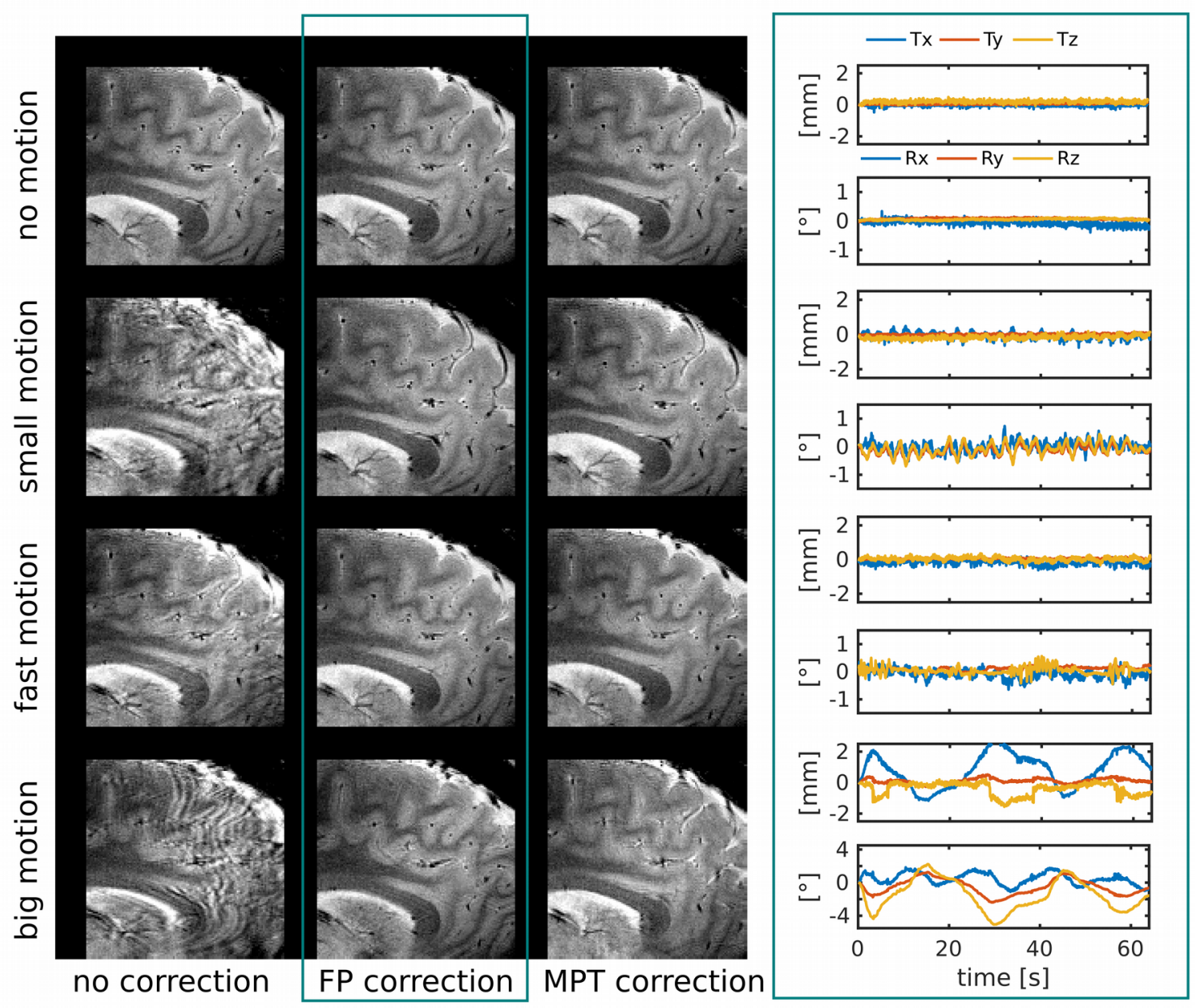

Figure 6: 2D Gradient echo images with an in-plane resolution of $0.5 \mathrm{~mm} \times 0.5 \mathrm{~mm}$ and a through-plane resolution of $1.6 \mathrm{~mm}$. Zoomed images for all twelve imaging conditions in one subject along with sample motion trajectories measured with the field probes for each motion condition. The full slice view of the measurements can be found in Supporting Figure S1. The motion trajectories for all twelve measurements are depicted in Supporting Figure S2. 

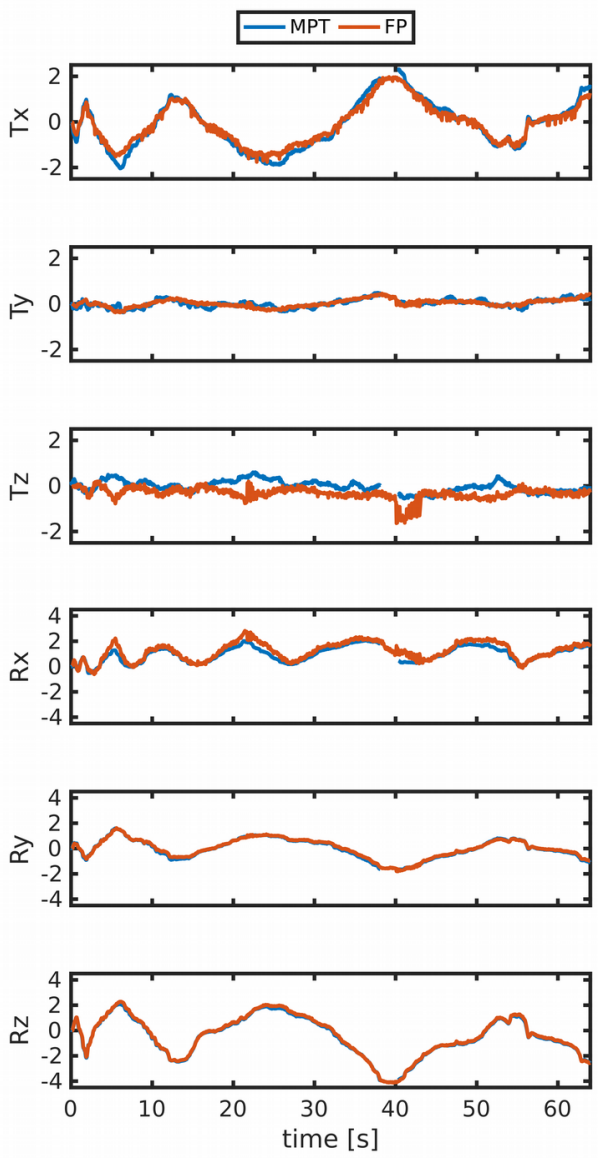

Figure 7: Motion trajectory for both tracking modalities from the 2D GRE measurement with big motion and field probe correction in Figure 6. 

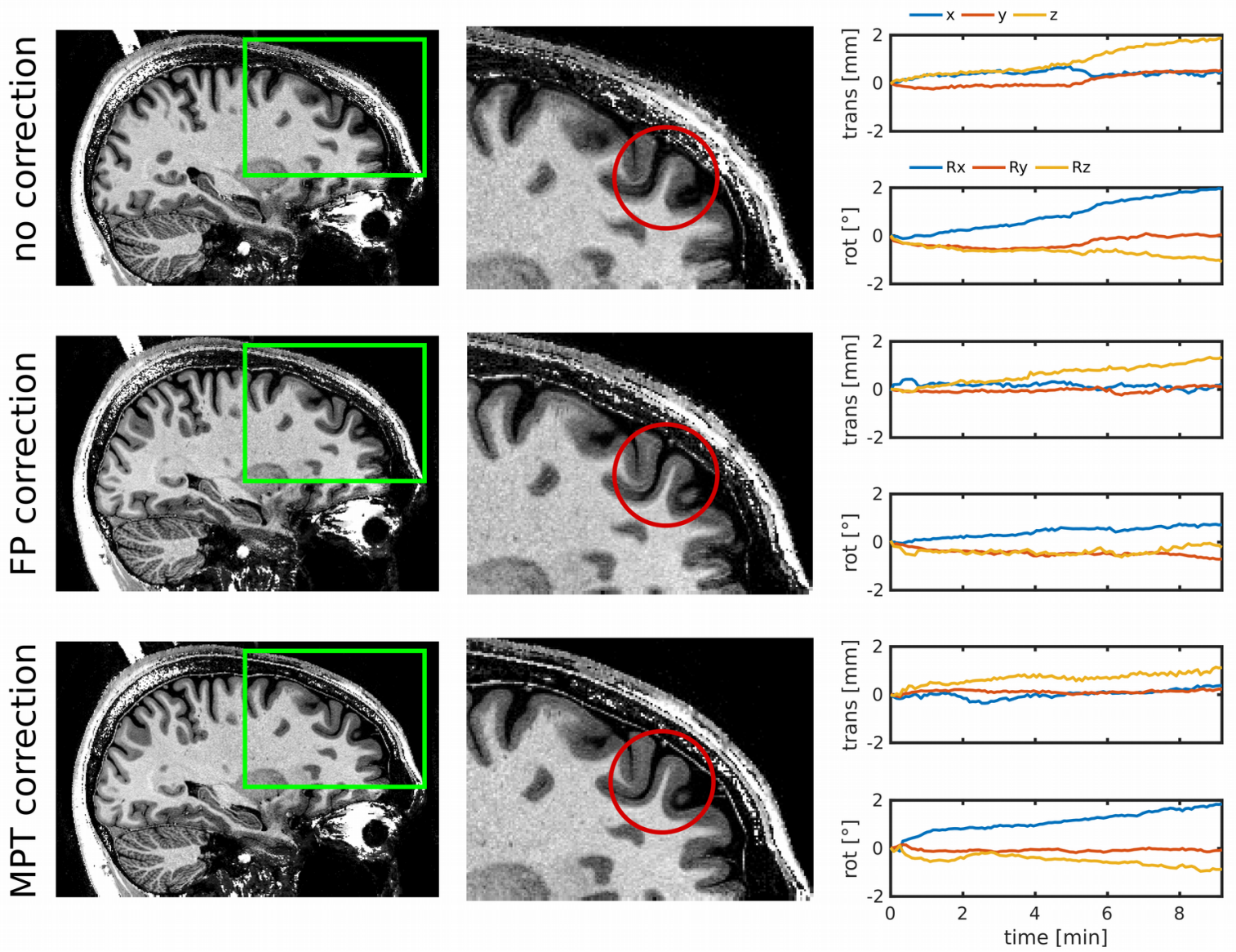

Figure 8: MP2RAGE $\left(0.5 \mathrm{~mm}^{3}\right.$ isotropic, acquisition time 9:03 $\mathrm{min}$ ) images for three motion correction conditions with corresponding motion trajectories acquired with the camera. 


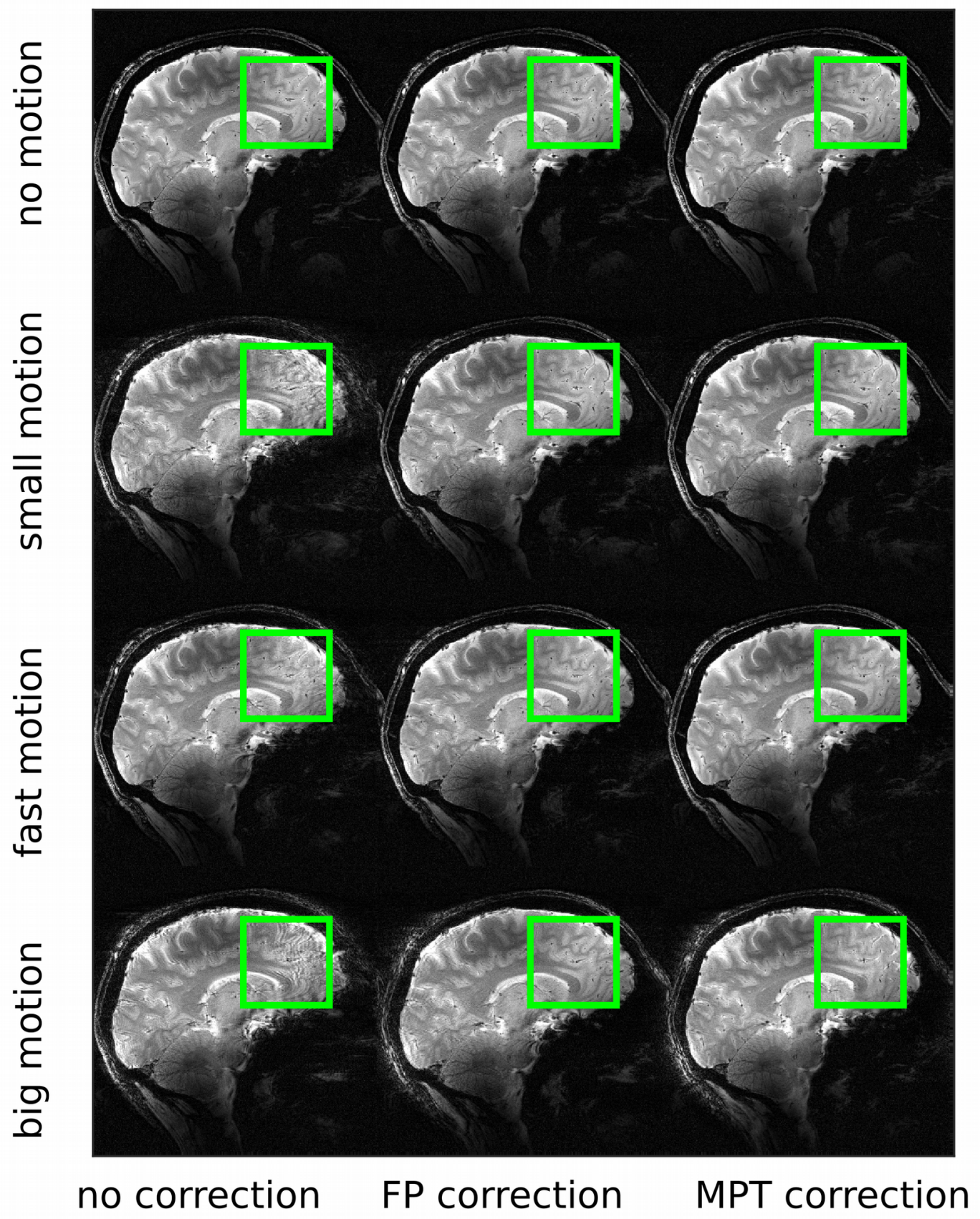

Supporting Figure S1: Full slice view for all twelve imaging conditions of the 2D GRE measurements in Fig. 6. 

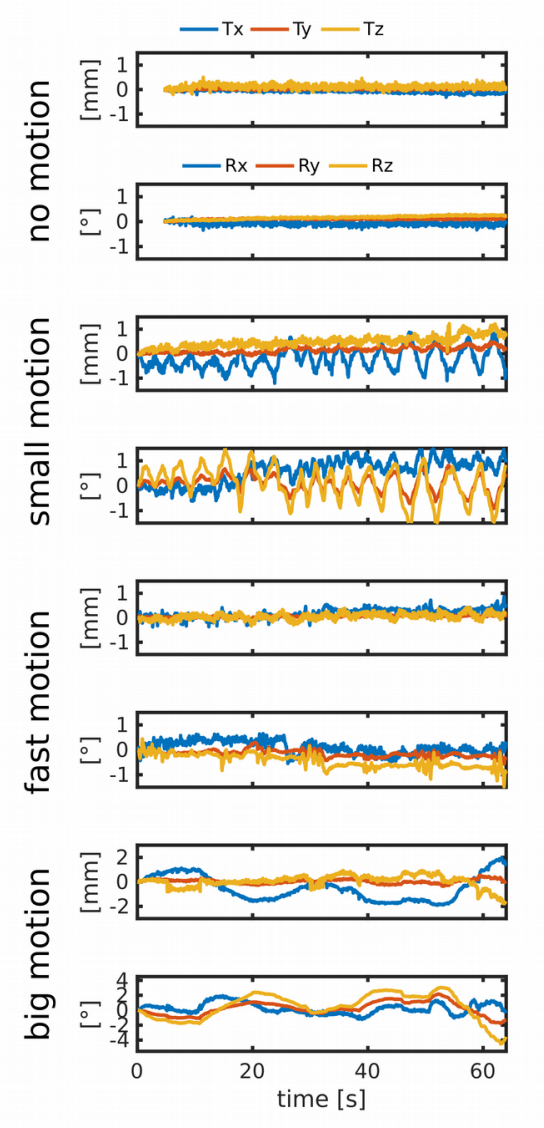

no correction
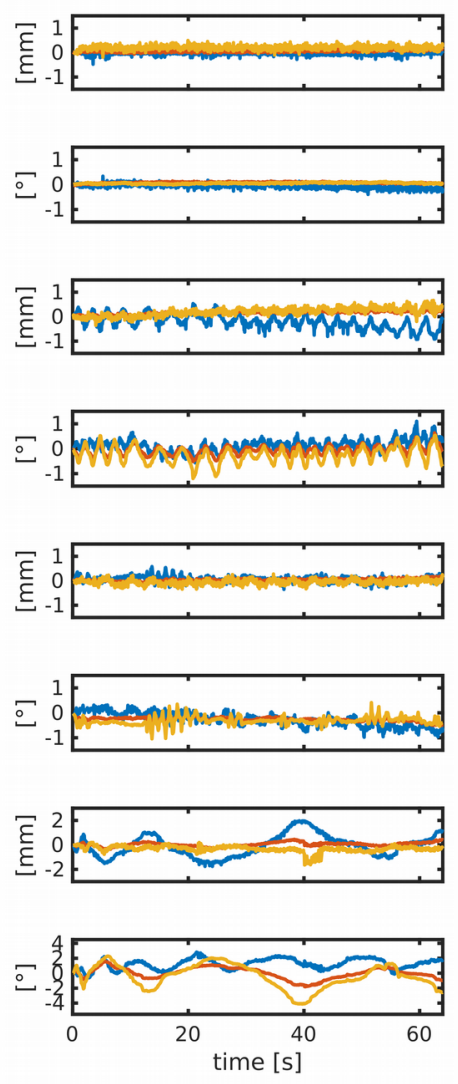

FP correction
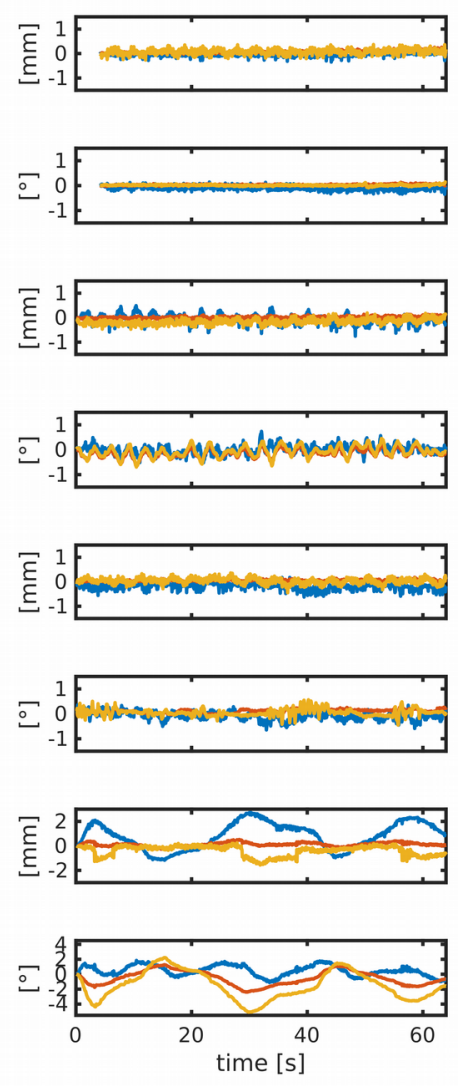

MPT correction

Supporting Figure S2: Motion trajectories captured by the field probes for all twelve motion and correction combinations for the 2D GRE measurements in Fig. 6. 